\title{
Strategies and Evolution in the Minority Game: A Multi- Round Strategy Experiment
}

\author{
Jona Linde $^{\mathrm{a}^{*}}$, Joep Sonnemans ${ }^{\mathrm{a}}$ and Jan Tuinstra ${ }^{\mathrm{a}}$ \\ a Amsterdam School of Economics, University of Amsterdam, \\ Roetersstraat 11, 1018 WB, Amsterdam, The Netherlands
}

February 28, 2013

\begin{abstract}
Minority games are a stylized description of strategic situations with both coordination and competition. These games are widely studied using either simulations or laboratory experiments. Simulations can show the dynamics of aggregate behavior, but the results of such simulations depend on the type of strategies used. So far experiments provided little guidance on the type of strategies people use because the set of possible strategies is very large. We therefore use a multi-round strategy method experiment to directly elicit people's strategies. Between rounds participants can adjust their strategy and test the performance of (possible) new strategies against strategies from the previous round. Strategies gathered in the experiment are subjected to an evolutionary competition. The strategies people use are very heterogeneous although aggregate outcomes resemble the symmetric Nash equilibrium. The strategies that survive evolutionary competition achieve much higher levels of coordination.
\end{abstract}

Keywords: minority game, strategy experiment, evolution, simulation, JEL codes: C63, C72, C91, D03

*Corresponding author, email address: j.linde@uva.nl, phonenumber: +31 205255902 


\section{Introduction}

In many situations the payoffs of your actions depend on the decisions of others facing exactly the same problem. A common feature on such occasions is that actions are strategic substitutes, i.e. an action taken by more people becomes less attractive. This occurs, for example, when firms need to choose whether to enter a new market, which (geographical) market to cater to, or whether to invest in a new technology. Other examples are traders deciding when to buy or sell a stock, commuters choosing a route and time to travel, workers deciding on union membership or high school graduates selecting a college program to enroll into. In such situations agents have to both coordinate and compete. Certainly if the agents are (nearly) symmetric, the payoffs for successful agents are large and there is no intuitive focal solution, one can imagine that coordination failure and instability can easily emerge

The "minority game" (Challet \& Zhang 1997) provides a very stylized, but intuitively appealing, way to model these types of problems. The minority game has an odd number of players who simultaneously have to decide between two options. Players who make the minority choice receive a reward, independent of the size of the minority, others receive no reward. Given the symmetry of the possible choices there is no reason to assume anything other than random choice in a one shot interaction. Interesting behavior may however emerge when players interact repeatedly, as they often do in the situations the minority game aims to model.

Even when the minority game is played repeatedly game theory provides only limited guidance. The game has many equilibria, none of which is focal. The pure strategy equilibria (any distribution of players across options leading to the largest possible minority) lead to very asymmetric payoffs and are non-strict. ${ }^{1}$ The symmetric mixed strategy Nash equilibrium (with each player choosing each option with equal probability) is, by definition also non-strict and may lead to small minorities, and hence suboptimal outcomes. All other equilibria are also non-strict and/or prone to suboptimal outcomes.

As game theory does not make any clear predictions about behavior in the minority game researchers have turned to simulation models and experiments to understand what happens in a repeated minority game. In simulation models different agents use different strategies to play the game repeatedly. A major drawback of this approach is that the strategies used in these simulation models are selected more or

\footnotetext{
${ }^{1}$ Players in the majority are indifferent between the two possible choices.
} 
less subjectively by the researchers. Whether decision makers would actually use those strategies is unclear. Since the choice of strategies is a crucial determinant of the dynamic behavior of the game this is highly unsatisfactory. Some studies allow strategies to evolve over time depending on their performance, but even in these models outcomes depend on the initial population of strategies and the types of strategies considered.

Laboratory experiments on the minority game can shed some light on aggregate outcomes, but, due to the large strategy space, it is impossible to distill the exact strategies used by the participants. As a result it is also impossible to study long term dynamics and the effect of evolutionary pressure on the population of strategies.

To solve this problem we use a strategy method experiment to elicit explicit strategies in a repeated (five-player) minority game. After gaining some experience with the minority game in the laboratory, participants program a strategy. A computer tournament between all submitted strategies then determines a ranking (the participants who submitted the five highest ranked strategies receive a monetary reward). After participants receive feedback on the performance of their strategy in the computer tournament they can revise their strategy for the next round. They can program new strategies and run simulations with these new strategies and strategies of others from the previous round, to evaluate the performance of programmed strategies. There are five rounds in total, each separated by a week.

We first analyze and classify the strategies submitted by the participants. Subsequently we run an evolutionary competition with all (107 unique) strategies to see which strategies survive. We find that the strategies submitted lead to aggregate outcomes that are comparable to those under the symmetric mixed strategy Nash equilibrium. However, individual strategies are very diverse, as is their performance. Nevertheless, there are some properties common to many strategies; in particular a majority of the strategies employs randomization, something excluded in many simulation studies. After evolutionary competition between the strategies four, relatively simple, strategies survive and coordination is enhanced substantially.

The remainder of the paper is organized as follows. In the next section we discuss the minority game and review the computational and experimental literature on this game. Section 3 discusses the design of the experiment. In section 4 we analyze the strategies submitted by the participants and in section 5 we use these 
strategies to establish which of them survives in an evolutionary competition. Section 6 summarizes the results.

\section{The minority game}

\subsection{Definition and relevance}

The minority game was introduced by Challet and Zhang (1997) as a stylized version of Arthur's famous El Farol bar game (1994). Arthur considers a population of 100 people deciding whether or not to visit the El Farol bar which will only be a pleasant experience if at most 60 people go. The minority game is a symmetric version of the El Farol bar game. There is an odd number of players $N$, who simultaneously have to choose one of two sides (say Red and Blue). All players that make the minority choice are rewarded with one 'point', the others earn nothing. More specifically, let $s_{i}=1$ when player $i$ chooses Red and $s_{i}=0$ when player $i$ chooses Blue. Payoffs for player $i$ are then given by

$$
\pi_{i}(s)=\left\{\begin{array}{ll}
s_{i} & \text { when } \sum_{j=1}^{N} s_{j} \leq \frac{N-1}{2} \\
1-s_{i} & \text { when } \sum_{j=1}^{N} s_{j} \geq \frac{N+1}{2}
\end{array} .\right.
$$

Note that the minority game is one of the simplest games one can think of: there are only two actions to choose from and only two possible payoffs. Furthermore, the game is symmetric.

The one-shot minority game has many Nash equilibria. Any action profile where exactly $\frac{N-1}{2}$ players choose one side constitutes a pure strategy Nash equilibrium. There are $\frac{N !}{\left(\frac{N+1}{2}\right) !\left(\frac{N-1}{2}\right) !}$ of such pure strategy Nash equilibria, which is a substantial number even for moderate values of $N$. There also exists a symmetric mixed strategy Nash equilibrium, where every player chooses Red with probability $1 / 2$. Finally, there are infinitely many asymmetric mixed strategy Nash equilibria. Take for example the profile where $(N-1) / 2$ players choose $R e d$ with certainty, $(N-1) / 2$ players choose Blue with certainty and the remaining player randomizes with any probability. 
Because of the plethora of equilibria and the symmetry of the minority game players in a one-shot version of this game can do little more than randomly choose one of the options. However, when the minority game is played repeatedly interesting behavior may emerge. Players could converge to one of the many equilibria but all equilibria are likely to be unstable. The pure strategy Nash equilibria lead to a very asymmetric distribution of payoffs, with otherwise identical players receiving different payoffs. ${ }^{2}$ Moreover, these equilibria are not strict: every player in the majority is indifferent between staying in the majority and unilaterally deviating to the minority, which then would become the majority choice. Players in the minority, foreseeing this, may preemptively switch. The mixed strategy equilibria are per definition also non-strict and moreover lead to inefficiencies. Expected aggregate payoffs are smaller than in a pure strategy Nash equilibrium, since there is a positive probability that the minority will be smaller than $(N-1) / 2 .{ }^{3}$ It is therefore unclear what type of behavior to expect in the repeated minority game.

Since its inception the minority game has received quite a lot of attention from physicists, but initially not so much from economists. ${ }^{4}$ There are several reasons for its popularity in physics. First, it is a simple game that allows for studying the interaction of heterogeneous agents as a complex adaptive system. Simulation methods and analytical tools from statistical physics (see e.g. Cavagna et al., 1999, Challet et al., 2000b) have been extensively applied to identify emergent macroscopic properties of these multi-agent systems. Secondly, it has been advanced as a stylized model of a financial market (see e.g. Challet et al. 2000a, 2001), and has become one of the canonical models in the field of 'econophysics'. In that interpretation the two sides of the minority game correspond to 'buying' and 'selling' a stock, respectively.

\footnotetext{
${ }^{2}$ Note that in the repeated minority game there exist pure strategy Nash equilibria where players rotate over the two options in such a way that every player spends the same number of periods in the minority. Total payoffs would then be the same for each player. However, in the absence of the possibility of communication, it seems very hard to coordinate on such an equilibrium, even if the number of players is relatively small. For a folk theorem on the infinitely repeated minority game see Renault et al. (2005).

${ }^{3}$ The equilibrium listed at the end of the previous paragraph is an exception to this but in that equilibrium the player who mixes always receives a low payoff so she has an incentive to disturb the equilibrium.

${ }^{4}$ For example, a search on Web of Knowledge (http://www.webofknowledge.com) gives more than 200 published articles with the phrase "minority game" in the title between 1998 and 2012. About 85\% of these articles have appeared in physics journals with the rest evenly spread between the fields of computer science, complex systems research and economics.
} 
If there are more (fewer) buyers than sellers, the price will be high (low) and sellers (buyers) make a profit.

Although the interpretation of the minority game as a model of a financial market may be criticized for being too simple, the minority game is closely related to, and a stylized representation of, many important economic problems. Congestion games for example (see Rosenthal, 1973, for a definition and Huberman and Lukose, 1997, for an application) are games where players make use of limited resources and payoffs are determined by how many other players use that resource. In fact, the minority game is a very simple example of a congestion problem with two routes and $N$ users, where each route has a capacity of exactly $(N-1) / 2$ users, and becomes fully congested when more than $(N-1) / 2$ users choose it.

Another problem closely related to the minority game is modeled by the market entry game. In such a game each of a number of $n$ firms has to decide independently and simultaneously whether to enter a (new) market or not. The payoff of entering depends upon the total number of firms entering and is typically linearly decreasing in that number, e.g. $\pi_{E}=k+r(c-m)$, where $c<n$ is the capacity of the market, $m$ is the number of entering firms and $k$ and $r$ are positive payoff parameters. Not entering gives payoffs of $\pi_{N}=k .{ }^{5}$ In a (pure or symmetric mixed strategy) Nash equilibrium (in expectation) between $c-1$ and $c$ firms will enter and in such an equilibrium the expected payoff difference between entering and not entering will be small or zero. ${ }^{6}$

On a more general level the minority game is an abstract version of games where actions are strategic substitutes. Well-known examples of such games are cobweb markets (Ezekiel, 1938) and Cournot oligopolies. For example, if most producers in a cobweb market predict next period's price to be higher than the rational expectations (RE) equilibrium price and therefore produce more than the RE

\footnotetext{
${ }^{5}$ Note that the El Farol bar game is in fact a special case of a market entry game, with a payoff function that does not linearly decrease in $m$ but is a step function with a discontinuity exactly when $m=c$. The payoff function of the El Farol bar game is flat everywhere else.

${ }^{6}$ There are some qualitative differences between the market entry / El Farol bar games on the one hand and minority / congestion games on the other. In the former there is always the safe option of not entering, whereas in the latter all alternatives are subject to strategic uncertainty with payoffs of every choice depending on the decisions made by the other agents. Another difference is that the pure strategy Nash equilibrium is Pareto efficient in minority and most congestion games, but not in the market entry game. Total payoffs in a market entry game increase when the number of entrants decreases below capacity.
} 
equilibrium quantity, the actual market clearing price will be lower than the RE equilibrium price. In such a cobweb market it is therefore better to disagree with the majority prediction. A similar argument holds for Cournot oligopolies. If the other firms on average have high production levels, it is optimal to supply a limited amount, and the other way around. The minority game is therefore a relevant, although stylized, model for a number of important economic problems.

\subsection{Strategies in the minority game: computational and experimental research}

In the physics literature the minority game is studied using computer simulations. In these simulations (see e.g. Challet and Zhang, 1997, 1998) the number of agents is large (typically between $N=101$ and $N=1001$ ) and every player has a fixed set of $S$ strategies (typically $S=2$, but sometimes higher values of $S$ are used), randomly drawn from the set of all strategies with memory $M$ (typically smaller than 10). Such a strategy maps the history of the past $M$ winning sides into a prediction of the next winning side. The number of different histories is therefore equal to $2^{M}$ and since any history can be mapped into one of two sides, the total number of different strategies is $2^{2^{M}}$, a number that increases fast with $M$ (e.g. for $M=5$ the total number of strategies is already about $\left.4.3 \times 10^{9}\right)$. Note that these strategies do not use information about the size of the minority and that they do not allow for randomization. ${ }^{7}$

Agents collect how well the strategies in their set predict the winning side (but do not consider the effect that a strategy they did not use might have had on the outcome) and in every period choose a side according to that strategy, from their set, that is the best predictor up to that period. ${ }^{8}$ Numerical simulations show that the number of agents choosing one side fluctuates around 50\%. The higher the volatility of fluctuations (implying that small minorities occur more often) the less efficient is the outcome.

One of the most celebrated results on the minority game is that of the dependence of the level of 'cooperation' on the parameter $\rho=2^{M} / N$, for the first time

\footnotetext{
7 An exception is Johnson et al. (1999). Cavagna et al. (1999) develop a continuous version of the minority game where instead of making a binary choice each agent submits a 'bid' that may lie somewhere in between the two extremes.

8 Alternatively, in some papers the choice of strategy is assumed to be probabilistic with the probability that a strategy is chosen positively related to its success, for example through a logit specification (see e.g. Cavagna et al., 1999, and Challet et al., 2000b).
} 
identified by Savit et al. (1999). For small values of $\rho$, where the number of agents is relatively large compared to the number of possible histories, aggregate behavior in the minority game is dominated by a cycle of period 2 and volatility is higher than under the symmetric mixed strategy Nash equilibrium. However, for moderate values of $\rho$, volatility drops below that of the symmetric mixed strategy Nash equilibrium, reaching a minimum value at some critical level $\rho=\rho^{c}$, and increasing towards volatility under the symmetric mixed strategy Nash equilibrium again, when $\rho$ increases beyond that critical value.

In the typical analysis of the minority game, as discussed above, each agent has a set of strategies from which it chooses one every period. The set of strategies of an individual agent is fixed and randomly drawn from the total set of strategies. ${ }^{9}$ It therefore contains arbitrary strategies, that may lack any rationale, but nevertheless the agent will hang on to these strategies forever.

A number of models have been advanced in which the set of strategies used evolves over time under evolutionary pressure (e.g. Li et al. (2000a, 2000b), Challet \& Zhang $(1997,1998)$ and Sysi-Aho et al. (2005). It turns out that this improves efficiency considerably - volatility is now always lower than under the symmetric mixed strategy Nash equilibrium, even for low values of $M-$ although volatility is still a non-monotonic function of $\rho$.

Clearly, aggregate behavior in minority games crucially depends upon the types of strategies agents are assumed to use. In the simulation studies discussed above the researchers had to make arbitrary assumptions, for example excluding the possibility of randomization. Results on a number of recent laboratory experiments on the minority game may help in understanding which strategies would actually be played by humans.

Platkowski and Ramsza (2003) and Bottazzi and Devetag (2003, 2007) performed experiments with the minority game and find that, although aggregate choices are volatile, (allocative) efficiency is higher than in the symmetric mixed

\footnotetext{
9 In some contributions an agent's strategies are not drawn independently from the set of all strategies. Challet et al. (2000b), for example, assume that an agent's second strategy is always chosen such that it is exactly opposite to its first strategy. Yip et al. (2003) consider strategies that are slightly biased to one alternative and show that this improves efficiency. Finally, Wang et al. (2009) consider a minority game with 'heterogeneous preferences', meaning that there are agents of different types $K$, with $K=0,1, \ldots, 2^{M}$, where an agent of type $K$ takes the first side for exactly $K / 2^{M}$ (randomly determined) histories.
} 
strategy Nash equilibrium. According to Bottazzi and Devetag participants seem to repeat their choices, particularly when they have more information and after a win. Moreover, participants seem to revert to pure strategies towards the end of the experiment.

In the experiments on the minority game discussed above it is impossible to determine definitively whether participants randomize or not. Chmura et al. (2010) therefore study a three-player minority game experiment where participants can explicitly use mixed strategies. Moreover, there is random re-matching of groups after each period, which makes the mixed strategy Nash equilibrium a more obvious candidate for individual behavior. They find that there is considerable heterogeneity in decision rules, and the behavior of only about a quarter of the participants is best described by the symmetric mixed strategy Nash equilibrium.

Devetag et al. (2011) consider a three-player minority game experiment where each player is represented by a team of three participants. Teams are video recorded and their discussion is analyzed to learn about strategies used for playing the minority game. Analysis of the video recordings reveals that teams rarely use a randomization strategy and that they tend to focus more on their own past behavior than on other teams over time, in particular when they have been successful. It is however unclear whether these strategies are affected by being taken by a group. For example the need to justify your proposal may well bias participants against randomization. ${ }^{10}$

Although these laboratory experiments shed some light on the type of strategies that people employ when playing the minority game, it is still difficult to infer exactly the strategies used. The main cause of this problem is the large strategy space when people can condition their action on a (long) history and randomize. A strategy experiment, where participants explicitly submit strategies to play the repeated minority game therefore seems appropriate. The strategy method has been applied before to related games, such as cobweb markets (Sonnemans et al., 2004), predictions in asset markets (Hommes et al., 2005), market entry games (Seale and Rapoport, 2000) and the El Farol bar game (Leady, 2000). Also the famous strategy tournament on the repeated prisoner's dilemma in Axelrod (1984) is related to our

\footnotetext{
10 Besides experiments with the minority game experiments have also been run with the related congestion (Iida et al. (1992) and Selten et al. (2007)) and market entry games (e.g. Sundali et al. (1995), Erev and Rapoport (1998), Rapoport et al. (1998) and Duffy and Hopkins (2005)). A robust finding from this literature is that aggregate behavior is roughly consistent with Nash equilibrium, but strategies vary widely at the individual level.
} 
work. In a recent paper Brandts and Charness (2011) provide an overview of experiments that directly compare the strategy method with the 'direct response' method. They find that in most studies these methods yield qualitatively similar results.

\section{Design}

We designed an experiment in which participants have to submit a strategy to play the five-player minority game for 100 periods. The experiment consists of five rounds, each separated by a week, and took place in April 2009. Participants are students of the so-called "beta-gamma" bachelor program, one of the most challenging programs of the University of Amsterdam. ${ }^{11}$

The first round of the experiment takes place in the CREED laboratory at the University of Amsterdam. The minority game is explained to the participants and they play the game two times for 10 periods in two different groups of players. After getting acquainted with the minority game by playing it, participants are explained on a handout and via the computer screen - how to formulate a strategy. They program two verbal strategies using the interface to check their understanding of formulating strategies, after which they formulate, test and submit their first strategy. ${ }^{12}$

After a few days all participants receive, by email, the results of the first round. From then on they can login on the website and try out new strategies against the population of strategies of the previous round. Within a week after the laboratory experiment they have to submit their new strategy (which could be identical to their old one) and fill out a short questionnaire. Two days after the deadline they receive the results of the second round. This procedure is repeated another three times. A week after the fifth and final round we explain the goals of the experiment in class, announce the results of the final round and pay out the earnings.

\footnotetext{
${ }^{11}$ These students follow courses in the natural sciences as well as the social sciences and they are typically well above average in motivation and capabilities. In particular, their programming experience is substantially higher than that of the average undergraduate student at the University of Amsterdam.

12 The participants can ask the experimenters for further instructions during the initial laboratory experiment. For the later rounds the experimenters were available for assistance via e-mail, although participants made no use of this possibility.
} 


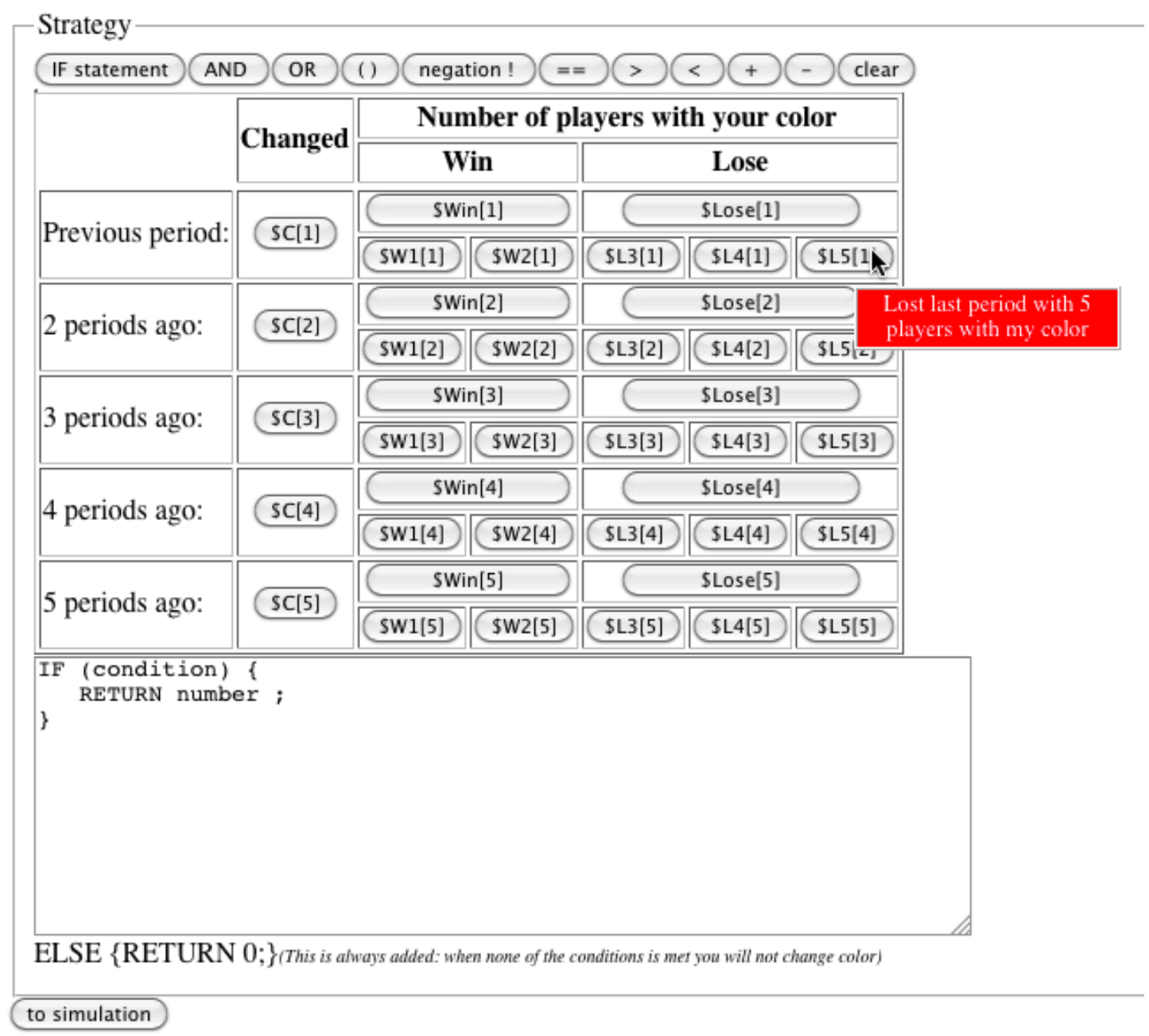

Figure 1: Computer screen as seen by the participants when they formulate a strategy.

\subsection{Formulating strategies}

Figure 1 shows the computer screen used by the participants to formulate their strategy. ${ }^{13}$ A strategy has the form of a list of IF-statements that (if the condition is met) return a number in the interval $[0,1]$, which is the probability of changing color. If the condition in an IF-statement is fulfilled, the subsequent IF-statements are ignored (the second and following IF-statements are treated like ELSE IF statements). If none of the conditions are met, the strategy returns 0 (i.e. no change of color). The number of IF-statements is unlimited and strategies can use logical expressions such as $\mathrm{AND}, \mathrm{OR}$, (in)equality and negation. In the instructions ample examples were given (see Appendix for an English translation of the instructions). The strategies can

\footnotetext{
13 The experiment is programmed in php/mysql and runs on a (Apache) web server. An English translation of the experiment can be found on www.creedexperiment.nl/minor/english and the interested reader is invited to formulate a strategy and run simulations with that strategy against actual strategies of our participants.
} 
use the history of the last 5 periods, which consists of the outcome in each of these previous periods (i.e. the size of the group that chooses the same color as the participant's strategy) and whether the strategy changed colors in that period or not.

We restricted the strategy space in two ways. First, as the minority game is a symmetric game where the labels of the two sides (red and blue) have no intrinsic meaning, we impose symmetry by letting strategies decide on changing color instead of choosing a color. Although individuals may have a preference for one of the colors, for example preferring winning when choosing blue over winning when choosing red, this limitation seems reasonable as using colors directly would double the number of variables per period. Second, we restrict the length of the history. We took this to be equal to five periods, which we believe gives a sufficient amount of flexibility for participants to develop strategies. ${ }^{14}$

Randomization and conditioning on the size of the group rather than merely winning or losing is explicitly allowed. The information about the last five periods is complete and contains whether the strategy made the winning decision, what the distribution of choices is and whether the strategy changed colors in that period. The total number of possible histories is therefore $5^{5} \times 2^{5}=100,000$.

\subsection{Simulations by participants}

A novel feature of our design, compared to other strategy method experiments, is that participants can run simulations with a strategy of their own making. Simulations are ran with four randomly drawn strategies (without replacement) from other participants from the previous round. ${ }^{15}$ Since strategies can use a history of up to 5 periods, first 5 random outcomes are drawn. After that, 100 periods are played according to the five strategies. After each simulation the results of the 100 periods, as well as those of the first five random periods are presented (see Appendix). In the presentation the choices of the other four strategies are sorted in each period (first the

\footnotetext{
${ }^{14}$ No more than $17 \%$ of all strategies submitted in our experiment uses information from 4 periods ago, and only $13 \%$ of the strategies uses information from 5 periods ago, whereas more than $90 \%$ uses information of the previous period, about two thirds of the strategies use information from two periods ago and about half of the strategies uses information from three periods ago. Limiting the history to five periods therefore seems to be relatively innocuous.

${ }^{15}$ In the first round no strategies from participants are available. The participants are informed that the strategies they compete against in the simulations they run in the first round are pre-programmed and are not necessarily similar to the strategies the other participants will submit. There are eleven preprogrammed strategies that do not condition on the history of outcomes and change with probability $q$, where $q=0,0.1,0.2, \ldots, 0.9,1$, respectively.
} 
red and then the blue choices) making it close to impossible to infer what the other strategies in the simulation are. In addition summary statistics are displayed for the 100 periods: the total number of points and the number of times the outcome was in category W1, W2, L3 L4 and L5, respectively, where W1 (W2) represents winning in a group of 1 (2) and L3 (L4, L5) represents losing in a group of $3(4,5)$. Participants can run as many simulations and try as many strategies as they want. They can use these simulations to see how successful their strategy is, but also to check whether their strategy behaves as they intended it to.

\subsection{Computer tournament, feedback and earnings}

After the deadline a computer tournament with all submitted strategies is run as follows. For every possible combination of five strategies a simulation of 100 periods is run (after five initial randomly selected outcomes), implying that the total number of simulations with about 40 strategies is around $10^{8}$. Subsequently we determine for each strategy the average number of points it earned in all the simulations it was involved in and use this to rank the strategies. The first five random outcomes in each simulation are not used in determining the average number of points. After each round, all participants receive an email with a ranking of the strategies and the average number of points earned by each strategy in the simulations. ${ }^{16}$ In addition they learn their earnings for that round. In the first four rounds the top five strategies receive $75,60,45,30$ and 15 euro, respectively. In the fifth and final round these amounts are doubled (and therefore are 150, 120, 90,60 and 30 euro). ${ }^{17}$

In addition in each round every participant who submits a strategy and fills out a short questionnaire receives 5 euro. One of the questions in the questionnaire is about how confident the participant is about the success of his/her strategy. To have an incentivized check on this question the opportunity is presented to wager the 5 euro. If a participant chooses to do so for a certain round, an extra reward is given when the strategy ends up in the top 5 of $75,60,45,30$ and 15 euro, respectively, in

\footnotetext{
${ }^{16}$ Strategies are identified by the nicknames of participants. It was not possible to observe the strategies used by other participants.

${ }^{17}$ It might be argued that due to this tournament incentive structure participants would not try to maximize their total points, but their relative ranking, resulting in different incentives than those implied by the minority game. However, there is an incentive for participants to maximize their number of points. Strategies that bear a cost in terms of points in order to do relatively well in one particular simulation by making the situation worse for the other four strategies in that simulation, will hurt their performance relative to the 30 to 35 strategies that are not present in that simulation.
} 
that round. Note that, with 45 participants, for a participant who believes her strategy is equally likely to end up in each of the 45 possible positions the expected value of this option is 5 euro. Therefore risk neutral participants who believe that the probability of their strategy reaching the winning positions is higher than that should wager their 5 euros.

\subsection{Questionnaire and web-server data.}

In the laboratory session we administered a short questionnaire about the background of the participants (like age, gender and programming experience). After submitting the strategy in each round a few questions about the (formulation of the) strategy are asked: how difficult it was to formulate the strategy, whether they had any problems with the formulation and how confident they are that the strategy will be successful. Finally, the incentivized question about confidence described above was asked.

\section{Results from the multi-round strategy experiment}

As explained above, in each round of the strategy experiment we run a simulation of 100 periods for each possible combination of five submitted strategies. The first round started with 42 participants submitting a strategy; in the subsequent rounds the number of submitted strategies was between 32 and 36. Average earnings for the whole experiment were $€ 58.70$ per participant, ranging from a minimum of $€ 0$ to a maximum of $€ 260$. In subsection 4.1 we present some results on the aggregate outcomes of the simulations, and in subsection 4.2 we take a closer look at individual strategies and categorize them with cluster analysis. 


\begin{tabular}{l|ccccc|cc}
\hline Round & 1 & 2 & 3 & 4 & 5 & $\begin{array}{c}\text { Symmetric } \\
\text { MSNE }\end{array}$ & PSNE \\
\hline $\begin{array}{l}\text { Number of } \\
\text { participants }\end{array}$ & 42 & 36 & 34 & 36 & 32 & & \\
\hline $\begin{array}{l}\text { Outcome } \\
\quad 3-2\end{array}$ & $63.90 \%$ & $61.66 \%$ & $64.57 \%$ & $66.07 \%$ & $64.32 \%$ & $62.50 \%$ & $100 \%$ \\
$\quad 4-1$ & $30.65 \%$ & $32.25 \%$ & $30.26 \%$ & $29.33 \%$ & $30.59 \%$ & $31.25 \%$ & $0 \%$ \\
$\quad 5-0$ & $5.45 \%$ & $6.08 \%$ & $5.17 \%$ & $4.60 \%$ & $5.09 \%$ & $6.25 \%$ & $0 \%$ \\
$\begin{array}{l}\text { Points } \\
\quad \begin{array}{l}\text { Average } \\
\quad \text { Standard Dev. }\end{array}\end{array}$ & 31.69 & 31.12 & 31.88 & 32.29 & 31.85 & 31.25 & 40 \\
$\quad \begin{array}{l}\text { Minimum } \\
\quad \text { Maximum }\end{array}$ & 29.31 & 21.65 & 24.98 & 28.00 & 18.93 & & \\
\hline $\begin{array}{l}\text { Average change } \\
\text { propensity }\end{array}$ & 34.68 & 41.65 & 36.96 & 39.87 & 43.06 & & \\
$\begin{array}{l}\text { Pearson correlation } \\
\text { change and points }\end{array}$ & -0.531 & 0.028 & -0.690 & -0.493 & -0.878 & & \\
\hline
\end{tabular}

Table 1: Distribution of outcomes and performance of participants over the rounds

\subsection{Aggregate outcomes and performance of participants}

For the repeated five-player minority game that we are considering the most efficient outcome is one where the minority consists of two players in every period. This happens in any pure strategy Nash equilibrium (PSNE). However, in the symmetric mixed strategy Nash equilibrium (MSNE), inefficiencies do occur since randomization implies that, with a positive probability, the minority will be smaller than two. In fact, it can be easily checked that in the symmetric MSNE the probability that a minority of two results is $62.50 \%$, whereas the probability of obtaining a minority of $1(0)$ is $31.25 \%(6.25 \%)$.

Rows 3 - 5 of Table 1 show the distribution of minorities resulting from the simulations with the submitted strategies. These distributions are very similar to those under the symmetric mixed strategy Nash equilibrium (seventh column of Table 1). Coordination of the strategies on larger minorities is slightly better than under the symmetric MSNE in most rounds but slightly worse in round 2. Clearly, coordination in any round is far from that obtained in a pure strategy Nash equilibrium (last column of Table 1).Table 1 also presents some results on the performance of the submitted strategies. Not surprisingly given the results on coordination presented in the previous paragraph the average number of points for the strategies is very close to individual performance in the symmetric MSNE. 
The symmetric MSNE clearly provides a good description of aggregate outcomes, but it performs poorly at the individual level. ${ }^{18}$ In the MSNE the payoffs of all strategies should be almost the same because most random variation would disappear when each strategy is involved in more than 30,000 simulations of 100 periods. The observed dispersion of payoffs generated by strategies, as measured either by the standard deviation of points, or by the range between the minimum and maximum number of points, is however considerable. ${ }^{19}$ These differences are therefore structural and there is substantial heterogeneity between the strategies in terms of their performance.

\begin{tabular}{lccccc|c}
\hline Round & 1 & 2 & 3 & 4 & 5 & Average \\
\hline Randomization & $83 \%$ & $83 \%$ & $71 \%$ & $69 \%$ & $69 \%$ & $75 \%$ \\
\# of periods considered & 2.43 & 2.36 & 2.47 & 2.47 & 2.31 & 2.41 \\
Consider winning situations & $86 \%$ & $94 \%$ & $82 \%$ & $75 \%$ & $78 \%$ & $83 \%$ \\
Consider losing situations & $88 \%$ & $89 \%$ & $85 \%$ & $78 \%$ & $81 \%$ & $84 \%$ \\
Consider whether you changed & $40 \%$ & $33 \%$ & $32 \%$ & $42 \%$ & $38 \%$ & $37 \%$ \\
\hline
\end{tabular}

Table 2: Characteristics of submitted strategies

Table 2 provides further evidence that individual strategies do not conform to the symmetric MSNE. As rows 4 and 5 show almost all strategies dependent on history. Winning and losing situations are explicitly included in the strategies about equally often. Whether the strategy changed is considered less often but is still taken into account by more than a third of the strategies. Many strategies use information about several rounds; on average 2.4 periods are considered, with almost all (94\%) strategies considering the previous period and very few (13\%) looking back 5 periods. A large majority of strategies is not a pure strategy but randomizes explicitly.

\footnotetext{
18 This is consistent with the findings in the experimental literature on congestion and market entry games discussed in footnote 10 .

${ }^{19}$ Although we simulated all possible combinations of strategies there is still some randomness in the average number of points, due to the first five random periods in each simulation and because strategies may be randomizing themselves. To check whether this randomness has an impact on the outcome we ran all simulations once more. This gives almost identical results: the correlation between the ranks in the two simulations turns out to lie between 0.998 and 1.
} 
Strategies are heterogeneous along many different dimensions. For example, the strategies vary from very short ("never change") to very long. ${ }^{20}$ They also differ substantially in how often they change colors. Some strategies never change colors, others change in about $95 \%$ of the periods. Change propensity is the only observed strategy characteristic that is significantly correlated with performance. In all rounds except for round 2 performance is negatively related to the propensity to change (Spearman rank correlation p-values smaller than 0.01). ${ }^{21}$ We can see no consistent decrease in this tendency over the rounds, which suggests that participants do not learn that their strategies change too often and adapt them accordingly.

Other measures strengthen the perception that participants did not manage to improve their strategy between rounds. We ran simulations with each participant's new strategy versus the other strategies from the previous round and vice versa. Even though participants could test their new strategy against the strategies of others from the previous period, in the majority of cases the old strategy would actually have done better against the old strategies of others than the new strategy (see Table 3). More important for the participants is the performance of their new strategy against the new strategies of others, but here the results is only slightly better. In only about $60 \%$ of the cases the new strategy does better than the old one would have done.

\begin{tabular}{l|cc}
\hline Round & $\begin{array}{c}\text { The new strategy would have done } \\
\text { better than the old strategy in the old } \\
\text { environment }\end{array}$ & $\begin{array}{c}\text { The new strategy does better } \\
\text { than the old strategy would have } \\
\text { done in the new environment }\end{array}$ \\
\hline 2 & $38.89 \%$ & $61.12 \%$ \\
3 & $53.57 \%$ & $44.11 \%$ \\
4 & $73.33 \%$ & $88.88 \%$ \\
5 & $23.33 \%$ & $46.87 \%$ \\
\hline Total & $46.77 \%$ & $60.87 \%$ \\
\hline
\end{tabular}

Table 3: The performance of the old and the new strategies in the old (column 2) and the new (column 3) environment

${ }^{20}$ One participant (participant 34) handed in strategies between 35 and $246 \mathrm{IF}$-statements in rounds 2 to 5. This participant ran about 5000 simulations with a 50\%-change strategy and determined, by means of a computer program, for each possible history what the optimal (non-random) response would be. Note that this strategy, although quite creative, responds to strategies from the previous round, not taking into account that those will change as well. This procedure has given rise to one successful strategy (the winner in round 3), but not in the other rounds partly because the strategies are untidy and prone to mistakes (the strategy for round 2, for example, contained a mistake and never changed). 21 This negative correlation between performance and the propensity to change is consistent with the results from computational models and laboratory experiments on the minority game (see Challet and Zhang, 1997, and Chmura and Pitz, 2006, respectively). 
Below we use strategy names to refer to strategies. The name of a strategy is the round in which it is first submitted, followed by the id number of the participant who submitted it, separated by a hyphen. For example, the strategy submitted by participant 40 in round 3 receives the name 3-40. Some strategies deserve special mention because they win a prize in several rounds: strategies 2-4 (in all four rounds in which it participated) and 3-11 (in all three rounds in which it participated). Also, strategy 1-10 - together with its slight variation 3-10 - wins a prize in three rounds. One could therefore argue that these are the three most successful strategies submitted during the experiment. Table 4 gives a verbal description of these strategies. In general, all of these successful strategies are reluctant to change, but make sure that they will not get stuck in a losing situation forever.

\begin{tabular}{|c|c|}
\hline $2-4$ & $\begin{array}{l}\text { If you changed two periods ago and lost two periods ago and you did not } \\
\text { change in the previous period and lost in the previous period, change with } \\
\text { probability } 1 \\
\text { Else if you lost in the last two periods in a group of three and you lost three } \\
\text { periods ago, change with probability } 0.8 \\
\text { Else if you lost in a group of } 4 \text { in the previous period and lost two periods } \\
\text { ago but did not change two periods ago, change with probability } 0.6\end{array}$ \\
\hline 3-11 & $\begin{array}{l}\text { Only change (with probability 1) when you lost in each of the last four } \\
\text { periods }\end{array}$ \\
\hline $\begin{array}{l}1-10 \\
(3-10)\end{array}$ & $\begin{array}{l}\text { If you lost in a group of } 3 \text { in the last two periods change with probability } 0.5 \\
\text { Else if you lost in a group of } 4 \text { in the last two periods, change with } \\
\text { probability } 1 \\
\text { Else if you lost in a group of five in the previous period, change with } \\
\text { probability } 0.5 \\
\text { Else if you lost in a group of } 4 \text { two periods ago and won in a group of } 2 \text { in } \\
\text { the previous period, change with probability } 0.75(0.5)\end{array}$ \\
\hline
\end{tabular}

Table 4: Description of strategies 2-4, 3-11 and 1-10 (3-10). Note that strategy 3-10 only varies slightly from strategy $1-10$ (both submitted by participant 10 ) in the probability of change in the last IF-statement ( 0.5 instead of 0.75$)$.

Besides the actual submitted strategies the experiment generates a wealth of webserver data, which may shed some light on how people try to learn. On average participants tried eight different strategies per round, and ran about 150 simulations with those strategies. More strategies were tried in the first two rounds and the average number of strategies tried stabilized at around five strategies per participant in each of the last three rounds. We also considered the login behavior of participants in rounds 2-5: on average they logged in between 1 and 2 times in each of those rounds, and were logged in almost two hours in total per round. 
The effects of these efforts on the rank of the participant are ambiguous, however. Both the number of strategies used and the number of simulations ran only have a positive effect upon the rank of the participant in round 4 (i.e. higher rank, Spearman rank correlation p-values of 0.0052 and 0.0021 , respectively). ${ }^{22}$

Remarkably, the number of strategies used has a negative effect on performance in round 2 (Spearman rank correlation p-value of 0.0105). There is no significant effect in any of the other rounds. The number of times logged on also has no significant effect in any of the rounds; the time logged on only has a negative effect on performance in, again, round 2 (Spearman rank correlation p-value of 0.0111). These results, combined with those from Table 3 , suggest that participants were on average not able to improve their strategies substantially.

An analysis of the decisions of participants to forego the fee of five euro in exchange for higher prizes suggests that participants were also unable to accurately predict the performance of their strategy. Participants choose higher prizes over the fee of five euro for about one third of all 180 submitted strategies. This decision was as to be expected - positively correlated with the answer to the question from the questionnaire how confident the participant was about his strategy in rounds 2, 3, 4 and 5 (Mann-Whitney test p-values $<0.002$ ), but remarkably only significantly positively correlated to the performance of the participant in round 4 (Mann-Whitney test p-value of 0.0088).

Given the limited amount of learning we take the pool of collected strategies as a sample of the type of strategies people will consider when first confronted with the minority game.

\subsection{Classification of strategies}

To get a better perspective on the kind of strategies submitted we performed a clusteranalysis on the 107 unique strategies. ${ }^{23}$ Figure 2 shows the resulting dendrogram. For

\footnotetext{
${ }^{22} \mathrm{We}$ also considered, in the spirit of the analysis presented in Table 3, the following. Let $S_{i}(R, T)$ be the score of the strategy that participant $i$ submitted for round $R$, when playing against the strategies the other participants submitted for round $T$. We checked whether the differences $S_{i}(R, R-1)-S_{i}(R-1, R-1)$ and $S_{i}(R, R)-S_{i}(R-1, R)$ are correlated with the number of simulations participants ran in that round. This is only (positively) significant for $S_{i}(3,2)-S_{i}(2,2)$, (p-value of 0.0059 ) suggesting that only in round 3 participants, on average, were able to improve their strategies, vis-à-vis those of the previous period, by running more simulations.

${ }^{23}$ Of the 110 unique strategies submitted we exclude the three very long computer generated strategies from participant 34 for the analysis in this and the next section (see footnote 20 for a discussion of those strategies). The main reason for this is that these are not the type of strategies that would
} 
this analysis we constructed a matrix of distances between strategies, calculated as follows. Strategies can use the history of the last 5 periods (outcome and whether they had changed colors in that period), which gives 100,000 possible histories. For every strategy the probability of changing color is calculated for each possible history. The distance between two strategies is then defined as the weighted average absolute difference between these probabilities. ${ }^{24}$ Because not all histories are equally likely (e.g. a 5-0 outcome is less likely than a 3-2 outcome) the weights are based upon the distribution that would results from the symmetric mixed strategy Nash equilibrium. ${ }^{25}$ We used the program "multidendrograms" 26 to draw the dendrograms, using the algorithm "joint between within" which both tries to minimize the distances within clusters and maximize the distances between clusters (Székely and Rizzo, 2005).

We find six clusters, labeled 1/6, 2/6 etc in Figure 2, and on a higher level three clusters, labeled $1 / 3,2 / 3$ and $3 / 3$. Table 5 displays for each of the six lower level clusters the most central strategy, that is, the strategy with the minimum average distance to the other strategies in that cluster. The most central strategies (CS) give some impression of the kind of strategies in that cluster. CS1/6 and CS2/6 change often, the first typically after losing in a period, the second independent of the history of outcomes. The central strategy from cluster $3, \mathrm{CS} 3 / 6$, is the one shot symmetric mixed strategy Nash equilibrium strategy (and therefore is also independent of the history of outcomes). CS4/6 seems to be a bit peculiar since it changes color after winning. However, this strategy might be quite sensible in an environment with many strategies that have the tendency to change after losing in the last period. ${ }^{27}$ In fact, CS4/6 is the strategy that wins the fifth round. CS5/6 is the very simple strategy of never changing and CS6/6 only changes (with probability 0.5 ) after losing in the previous period.

\footnotetext{
typically be used by human decision makers and they are difficult to interpret (another minor practical reason is that these strategies increase computation time considerably).

${ }^{24}$ This is a continuous version of the Hamming distance.

${ }^{25}$ As discussed in Section 4.1 the symmetric MSNE leads to the outcomes 5-0 in 6.25\%, 4-1 in 31.25\% and $3-2$ in $62.5 \%$ of the periods. This is very close to the numbers in the experiment (see Table 1 ) and in the simulation discussed below $(5.63 \%, 30.87 \%$ and $63.50 \%$, respectively).

${ }^{26} \mathrm{http}: / /$ deim.urv.cat/ sgomez/multidendrograms.php, see also Fernández and Gómez (2008).

${ }^{27}$ In the terminology of Selten et al. (2007) strategy CS4/6 can be classified as using a "contrary response mode', whereas for example strategy CS1/6 uses a 'direct response mode'.
} 


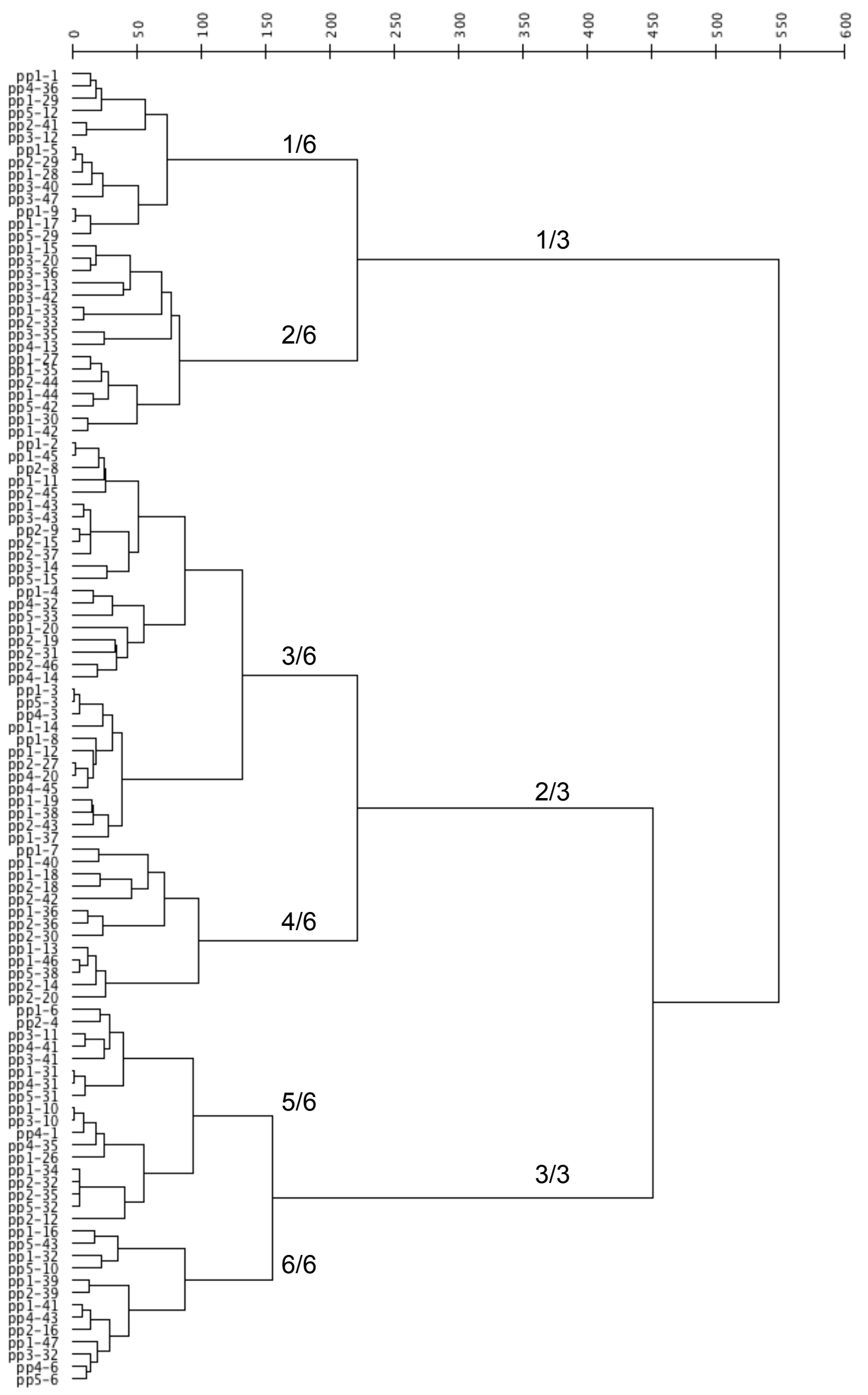

Figure 2: A cluster analysis of the 107 unique strategies. 


\begin{tabular}{|c|c|}
\hline Cluster & Most central strategy \\
\hline $1 / 6$ & $\begin{array}{l}1-5 \\
\text { If you have lost the last period change with probability } 0.8 \text {; if you win three } \\
\text { times in a row change with probability } 0.5 \\
3-36 \\
\text { Always change with probability } 0.9\end{array}$ \\
\hline $3 / 6$ & $\begin{array}{l}2-27 \\
\text { Always change with probability } 0.5 \\
1-46 \\
\text { Only change when you won the last period. }\end{array}$ \\
\hline $5 / 6$ & $\begin{array}{l}1-34 \\
\text { Never change } \\
4-6 \\
\text { When you lost the last period change with probability } 0.5 \text {. }\end{array}$ \\
\hline
\end{tabular}

Table 5: The most central strategy in each of the six clusters (the strategy with the minimum average distance to the other strategies in that cluster)

To further study the differences between the clusters we ran 500,000 simulations of a 100-period minority game where, for each simulation, we randomly selected (with replacement) five strategies from the set of 107 unique strategies. For all strategies the average number of points over these simulations and the percentage of changes in these simulations are calculated. The second column of Table 6 confirms what is suggested by the central strategies listed in Table 5: that strategies from clusters 1 and 2 change relatively often, strategies from cluster 5 and 6 relatively little, and strategies from cluster 3 and 4 somewhere in between. ${ }^{28}$ Furthermore, the strategies in clusters $5 / 6$ and 6/6 earn more points than the strategies in the other clusters (Mann-Whitney tests, all $p$-values $<0.001$ ) and strategies in cluster $5 / 6$ perform better than those in cluster $6 / 6$ (p-value $<0.001)$.

We have ran additional simulations to understand the behavior of strategies in the different clusters. ${ }^{29}$ We ran simulations in a fully homogeneous setting, i.e. with five identical strategies and simulations where all strategies were selected from the same cluster. The fourth and fifth columns of Table 6 show the averages per cluster. Especially in purely homogeneous settings (column four) average earnings in each cluster are very low, even substantially lower than in the symmetric mixed strategy

\footnotetext{
${ }^{28}$ All differences are statistically significant with the exception of $4 / 6$ versus $1 / 6$ and 3/6.

${ }^{29} \mathrm{We}$ also studied whether the clusters differ in other aspects, like complexity, length of history used, etc, but found no consistent differences.
} 
Nash equilibrium. This suggests that participants designed their strategies to either exploit other strategies or cooperate with them. ${ }^{30}$

Both the completely homogeneous and the within cluster simulations show that strategies from clusters $1 / 6$ and 4/6 are particularly badly equipped to play against strategies that are similar to them. Strategies from clusters $2 / 6,5 / 6$ and $6 / 6$ perform better than the strategies from the other clusters (who on average earn less than they would in the symmetric mixed strategy Nash equilibrium). Strategies from cluster 5/6 obtain even more point than when playing the full population of strategies; although even they perform worse than a purely random strategy in purely homogeneous settings.

\begin{tabular}{c|cccccc}
\hline Cluster & $\begin{array}{c}\text { Change } \\
(\text { Sd })\end{array}$ & $\begin{array}{c}\text { Points } \\
(\text { Sd })\end{array}$ & $\begin{array}{c}\text { Earnings in } \\
\text { homogeneous } \\
\text { simulations } \\
(\text { Sd) }\end{array}$ & $\begin{array}{c}\text { Earnings in } \\
\text { simulations } \\
\text { within cluster } \\
(\text { Sd) }\end{array}$ & $\begin{array}{c}\text { Evolution } \\
\text { last } \\
\text { generation } \\
\text { alive }(S d)\end{array}$ & $N$ \\
\hline \multirow{2}{*}{$1 / 6$} & 54.20 & 31.20 & 16.28 & 20.47 & 11.27 & 14 \\
& $(14.83)$ & $(1.74)$ & $(10.71)$ & $(12.97)$ & $(1.91)$ & \\
$2 / 6$ & 71.34 & 30.33 & 27.97 & 32.79 & 9.89 & 16 \\
& $(9.23)$ & $(1.16)$ & $(7.91)$ & $(4.70)$ & $(1.23)$ & \\
$3 / 6$ & 44.66 & 30.70 & 23.95 & 30.31 & 11.44 & 33 \\
& $(12.06)$ & $(0.59)$ & $(9.05)$ & $(2.64)$ & $(0.74)$ & \\
$4 / 6$ & 46.43 & 30.80 & 12.94 & 23.89 & 11.72 & 13 \\
& $(15.28)$ & $(1.28)$ & $(9.68)$ & $(9.63)$ & $(0.89)$ & \\
$5 / 6$ & 17.00 & 34.46 & 27.59 & 36.60 & 105.42 & 18 \\
& $(8.18)$ & $(1.69)$ & $(9.25)$ & $(3.90)$ & $(161.36)$ & \\
$6 / 6$ & 33.99 & 32.58 & 25.54 & 32.38 & 95.74 & 13 \\
& $(10.22)$ & $(1.23)$ & $(11.64)$ & $(3.02)$ & $(179.97)$ & 13 \\
\hline \multirow{2}{*}{ Tot } & 44.16 & 31.59 & 23.02 & 29.92 & 37.27 & \multirow{2}{*}{107} \\
& $(19.88)$ & $(1.89)$ & $(10.68)$ & $(8.11)$ & $(97.68)$ & \\
K-W & 0.000 & 0.000 & 0.000 & 0.000 & 0.000 & \\
\hline
\end{tabular}

Table 6: Column 2 and 3 display the percentage change and the average number of points per cluster in a simulation with all 107 unique strategies. Column 4 displays the average points in homogenous groups ( 5 identical strategies) and column 5 displays the average number of points in groups with only strategies from the same cluster. Column 6 displays the average last generation alive in an evolutionary simulation (see Section 5 for a discussion). The last row gives Kruskal-Wallis tests (p-values based on 2-sided tests).

\footnotetext{
${ }^{30}$ For an extreme example take the central strategy of cluster 4/6 (1-46) "Change only when you won the last period" (the winner of the final round in the experiment). After the first period this strategy will lose every round in a homogeneous group. Note however that in the experiment strategies could only meet exact copies of themselves if other participants would submit the same strategy.
} 


\section{Evolutionary competition between submitted strategies}

In this section we consider an evolutionary competition between the strategies submitted by the participants to determine which kinds of strategy survives. If we see the set of strategies gathered as representative of the strategies people consider when faced with the minority game the evolutionary competition shows which of these strategies survive in the long run.

We model the evolutionary competition between strategies as follows. In the first generation every strategy $i$ has the same weight $w(i, 1)=\frac{1}{N}=\frac{1}{107}$. In every generation $g$ we run 2000 simulations of 100-period minority games. In each of these games five strategies are randomly selected (with replacement), where the probability of selecting strategy $i$ equals its weight $w(i, g)$. For each strategy $i$ we determine the average number of points it earned, averaged over all simulations in a generation that it was part of. We denote this average by $P(i, g)$. We also determine the average number of points earned by all strategies, averaged over all simulations, and denote this by $M(g)$. After each generation of 2000 simulations the weights of the different strategies are updated on the basis of how well they did as compared to the whole population of strategies. This updating is formalized as follows:

$$
\bar{w}(i, g+1)=\max ((1+\lambda[P(i, g)-M(i, g)]) w(g, i), 0)
$$

where $\lambda$ is a positive parameter which measures selection pressure. Note that if a strategy performs better than the average strategy in a generation, its weight increases $(\bar{w}(i, g+1)>w(g, i))$. If a strategy performs much worse than average, its weight is set to $\bar{w}(i, g+1)=0$ and the strategy becomes extinct. The same thing happens if a strategy was selected in none of the 2000 simulations of a generation (which is only likely to happen if its weight is very small to begin with). The new weights are then determined as

$$
w(i, g+1)=\frac{\bar{w}(i, g+1)}{\sum_{j} \bar{w}(j, g+1)}
$$

to make sure that the weights sum up to one again. 


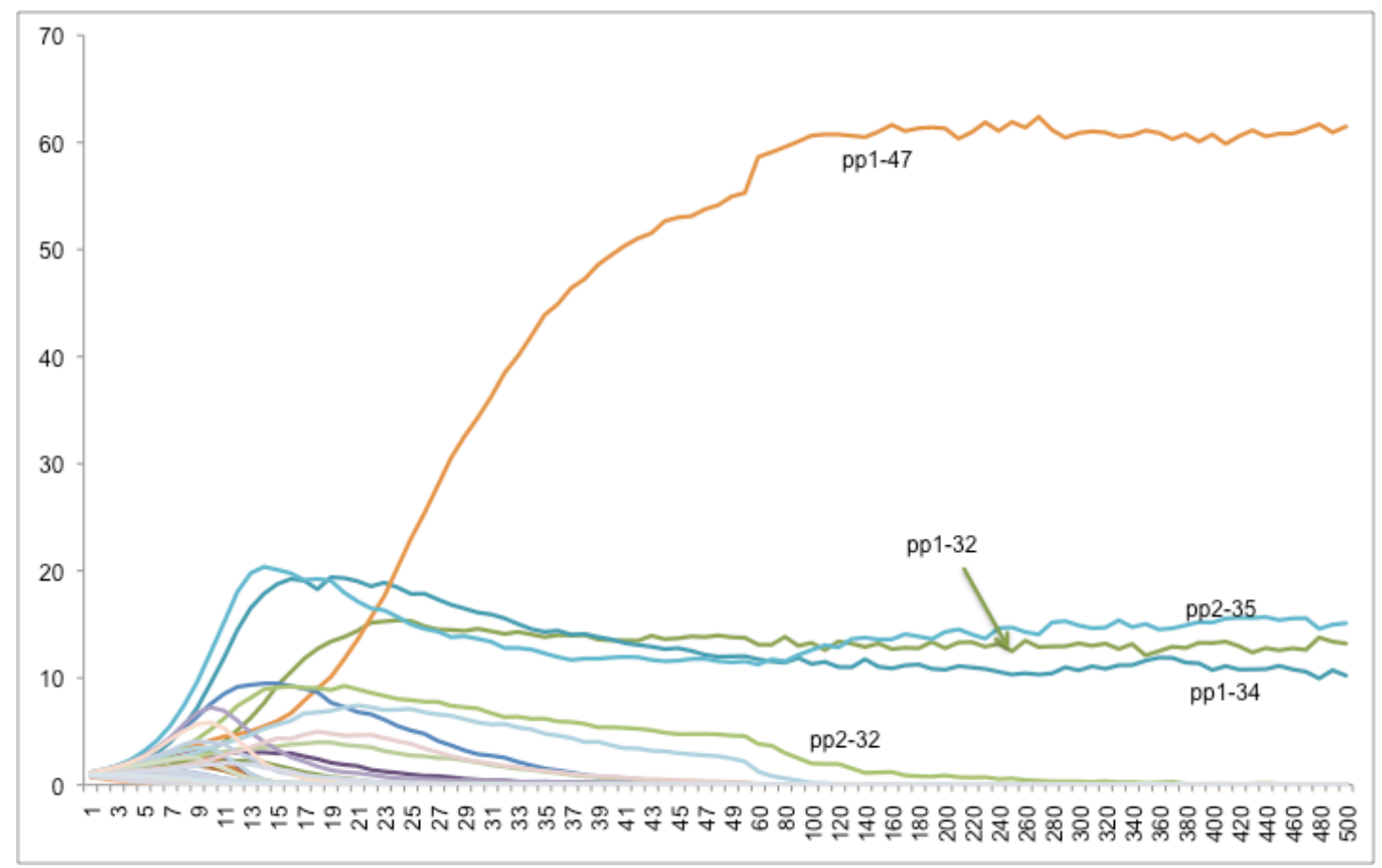

Figure 4: Evolutionary analyses with a starting population of 107 strategies. On the horizontal axis the generation (after generation 50 in steps of 10), on the vertical axis the percentage of the population, averaged over five evolutionary simulations.

We ran this evolutionary simulation five different times with the same 107 strategies and for 500 generations, with the parameter $\lambda$ set equal to $0.05 .^{31}$ Figure 4 shows how the weight of each of the 107 strategies, averaged over the five simulations, evolves over the 500 generations. ${ }^{32}$ Clearly there are four strategies which manage to survive.

\footnotetext{
${ }^{31} \mathrm{~A}$ low value of $\lambda$ leads to a very slow evolution and long simulation times, while a large value increases the role of 'bad luck'. The parameter value we chose is relatively low: even the worst performing strategies take at least 8 generations to die out.

32 The outcome of this evolutionary simulation is very robust: in all five simulations the same four strategies survive for 500 generations with more or less the same weights in the last generation. In only one of the simulations another strategy (2-32) survives for the first 500 generations, but its weight in generation 500 is very small $(0.11 \%$ of the population), and it seems likely that this strategy would die out if the evolutionary simulation would run longer.
} 


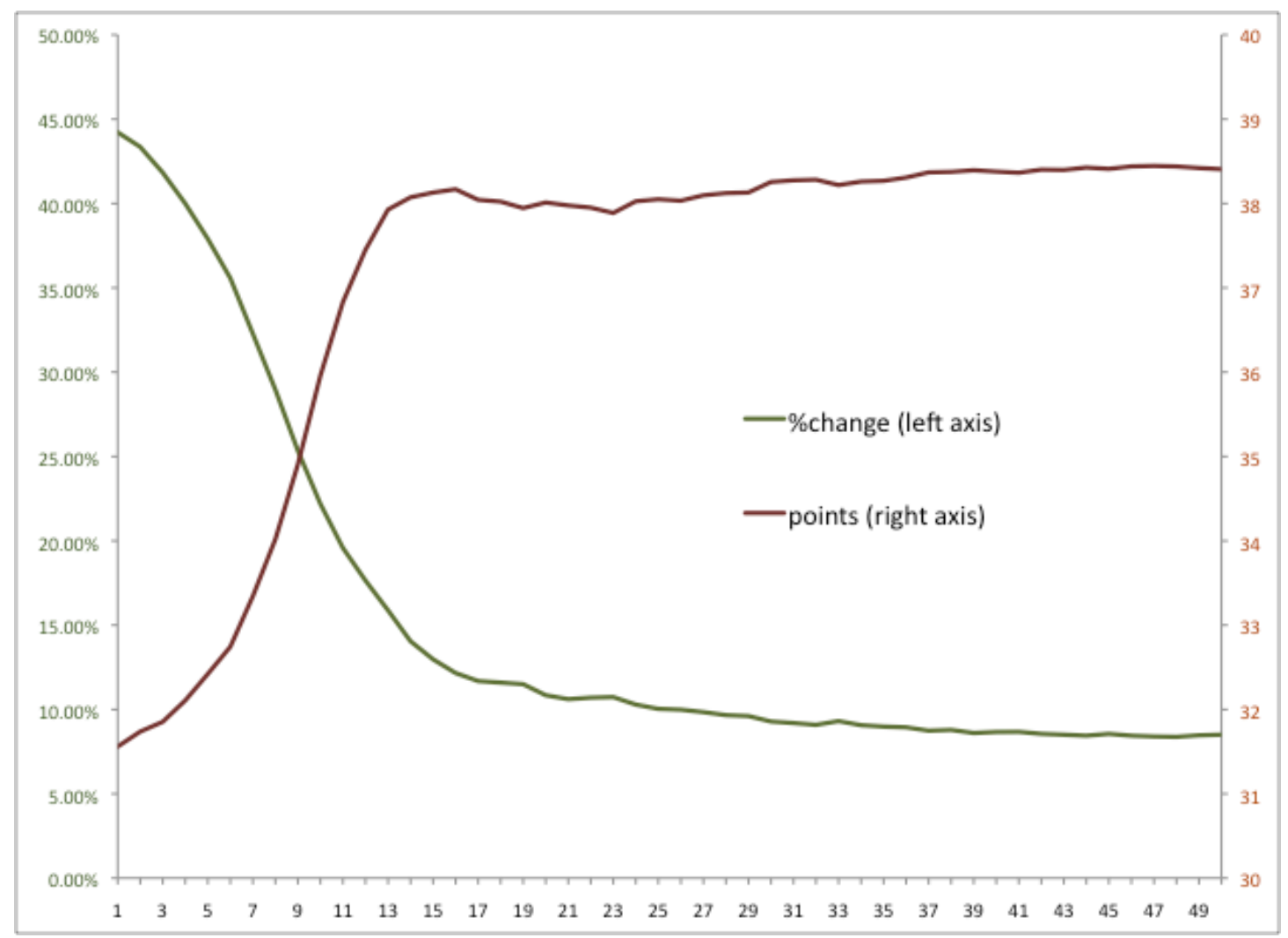

Figure 5: Generations are on the horizontal axis, the left vertical axis (green line) is the average percentage that a strategy changes and the right vertical axis (red line) the average number of points of a strategy in a game of 100 periods, again averaged over the five evolutionary simulations.

Before we consider the surviving strategies we examine the effect of the evolutionary simulation on two important population characteristics: the average number of points attained and the propensity to change color. Figure 5 shows, for the first 50 generations, the average number of points and the average percentage that strategies in the population change color (these levels remain more or less constant after the first 50 generations). The most striking result of the evolutionary simulations is the rapid increase in the average number of points, which increases from about 31.5 (very close to the average number of points under the symmetric mixed strategy Nash equilibrium, 31.25) to 38.5 (which approaches the maximal possible average number of points that is obtained under any pure strategy Nash equilibrium, 40). After 50 generations the efficient distribution (with two players in the minority) occurs in $92.66 \%$ of the periods, whereas an inefficient outcome with one (zero) player in the minority occurs in $6.97 \%(0.37 \%)$ of the periods, and these numbers remain more or less constant from then on. ${ }^{33}$

\footnotetext{
${ }^{33}$ For the first generation these numbers are $63.63 \%, 30.85 \%$ and $5.52 \%$.
} 
Figure 5 suggests that the substantial increase in coordination is directly related to a decrease in the percentage with which strategies in the population change colors, which goes from about $45 \%$ when all 107 strategies are present in the population, to around $8 \%$ when only the four survivors remain. This relationship between change and number of points may be caused by the fact that predictability, and thereby coordination, increases in an environment with strategies that rarely change, provided that at least some of these strategies condition on the history in a way that corrects inefficient situations.

\begin{tabular}{ll}
\hline $1-47$ & If you won the last period: don't change \\
& Else if you lost the last period in a group of 3 or 4 : change with probability \\
& 0.2 \\
& Else if you lost one or both of the periods -2 and -3 : change with probability \\
& 0.6 \\
\hline $2-35$ & Only change (with probability 1) when you lost the last period in a group of 5 \\
\hline $1-32$ & $\begin{array}{l}\text { Only change (with probability 1) when you lost the last period in a group of } 4 \\
\text { and/or if you won in period }-3 \text { in a group of } 1 .\end{array}$ \\
\hline $1-34$ & Never change
\end{tabular}

Table 7: The four surviving strategies after 500 generations.

The surviving strategies, listed in Table 7, indeed chance very little and, taken together, avoid getting stuck in inefficient situations. Strategy 2-35 breaks a situation where all strategies choose the same color and 1-47 and 1-32 situations where 4 strategies choose the same color. In contrast, 1-34 is more conservative and never changes. Except for 1-47 these strategies do not disturb an efficient (minority of 2) outcome, even if they lose.

As Table 8 shows, strategy 1-34 earns less than the other surviving strategies in the last generations, which indicates it is still decreasing in strength. We have run simulations for all possible group compositions with the four survivors and solved for the population equilibrium by finding a distribution for which all active strategies earn the same average number of points (this equilibrium is given in the sixth column of Table 8). We find that in this equilibrium strategy 1-34 is not present and the population fraction of strategy 2-35 is higher than after the evolutionary simulation. Because the only difference between these two strategies occurs when all strategies pick the same color, which occurs only $0.37 \%$ of the time, there is little evolutionary pressure on strategy 1-34. The average number of points earned in the population equilibrium is slightly higher than that in generation 500 (38.47 versus 38.40 ). 
The fifth column of Table 8 presents the earnings of the surviving strategies in a population where they only meet themselves. A striking observation is that, except for strategy 1-47, all strategies do poorly in such a situation, because these strategies can all get stuck in an inefficient losing situation. In contrast strategy 1-47, the clear "winner" of the evolutionary competition, with a proportion of the population of more than $60 \%$, changes when it is on the losing side for too long, independent of whether it is an efficient or inefficient situation. Although this strategy does very well in a homogeneous setting it performed relatively poorly, compared to the other surviving strategies, as long as there are still many other strategies in the population (see Figure 4). Nevertheless it did reach fifth place in round 1 of the experiment.

\begin{tabular}{l|c|ccccc}
\hline Strategy & Cluster & $\begin{array}{c}\text { Population } \\
\text { proportion } \\
\text { after 500 }\end{array}$ & Earnings & $\begin{array}{c}\text { Earnings } \\
\text { Homogeneous }\end{array}$ & $\begin{array}{c}\text { Population } \\
\text { equilibrium }\end{array}$ & $\begin{array}{c}\% \\
\text { Change }\end{array}$ \\
\hline $1-47$ & $6 / 6$ & $61.5 \%$ & 38.43 & 36.8 & $60.1 \%$ & $14.8 \%$ \\
$2-35$ & $5 / 6$ & $14.6 \%$ & 38.98 & 31.3 & $24.5 \%$ & $4.1 \%$ \\
$1-32$ & $6 / 6$ & $13.5 \%$ & 38.24 & 21.7 & $15.4 \%$ & $28.8 \%$ \\
$1-34$ & $5 / 6$ & $10.4 \%$ & 37.76 & 31.3 & $0 \%$ & $0 \%$ \\
Overall & & & 38.40 & & & \\
\hline
\end{tabular}

Table 8: Characteristics of the four surviving strategies. Cluster refers to Section 4.2 and Figure 2. The population proportion (earnings) are the average in generations 491-500 in the 5 evolutionary simulations. The earnings solo are the average earnings of that strategy in homogenous groups. The population equilibrium displays the population proportions in the equilibrium is which all strategies have the same expected earnings (38.47). The final column displays the percentage of periods the strategy changes color.

An interesting observation is that except for strategy 1-47 none of the surviving strategies did very well in the experiment. Conversely, most strategies that won a price become extinct within the first 20 generations (with the winners from clusters $1 / 6$ and $4 / 6$ dying out first). Some of the most successful strategies in the experiment manage to survive somewhat longer. Apparently these strategies perform well because they can exploit other strategies but when those exploitable strategies disappear they are overtaken by the strategies that eventually win the evolutionary competition.

To explore whether strategies indeed exploit other strategies we examine their effect on other strategies' performance in the experiment. Specifically we compare the average number of points scored by strategies submitted in the same round in 
simulations where a particular strategy is present to the average number of points scored by the other strategies in all simulations in that round. ${ }^{34}$ This difference measures the effect of a strategy on the performance of other strategies. If other strategies on average perform worse (better) when a particular strategy is present than when it is not present we classify that strategy as exploitative (cooperative). ${ }^{35}$ All strategies that attract more than $4 \%$ of the population at some point during the evolutionary simulation are classified as exploitative, with the exception of strategies 1-32 and 2-35. Both of these strategies are survivors in the experiment, but by a different route.

Strategy 2-35 manages to be successful from the beginning of the experiment, despite on average helping other strategies. The reason appears to be its reluctance to change which is very successful in the full population of strategies as can be seen from the early rise of strategy 1-34 which never changes. Strategy 1-32 does not do very well in the beginning but comes into its own later on when strategies that change too often have died out. In a population where strategies change less often the fact that 1-32 changes color to prevent getting stuck in a 4-1 situation helps increase efficiency. All strategies that earn a prize in the experiment are also exploitative. These strategies can only be successful when the strategies from which they profit are present.

To test robustness we ran 25 additional evolutionary simulations, each with a random selection of 50 of the original 107 strategies. ${ }^{36}$ In these simulations between 2 and 7 strategies survive up to generation 500. In 24 of the 25 simulations all survivors are from the clusters $5 / 6$ and $6 / 6$, the exception is the only one with 7 survivors where there is one survivor from cluster $3 / 6$ and one from cluster $4 / 6$, with the other five from clusters $5 / 6$ and $6 / 6$. The four surviving strategies from the original simulation all do very well: 1-47, 1-32 and 1-34 are survivors in all simulations in which they are

\footnotetext{
${ }^{34}$ If a strategy is present in more than one round we take the average effect on the points scored by the other strategies over all rounds in which the strategy is present.

35 Note that this measure tests whether a strategy is cooperative or exploitative relative to the other strategies in the round(s) where it is present, not whether strategies in a round are on average cooperative or exploitative. (E.g. if all strategies in a round help others to the same extent none of them will be classified as cooperative.)

${ }^{36}$ The only other difference with the original evolutionary simulations is that now in a generation only 1000 , instead of 2000 minority games (each of 100 periods) are simulated. Since the number of strategies is less than half the number for the original evolutionary simulation, this implies that (at least in the beginning of the evolutionary simulation, when all weights are equal) each strategy is involved on average in the same number of simulations as in the original evolutionary simulation.
} 
involved; $2-35$ survives in 10 of the 13 (77\%) simulations in which it is involved. All other strategies perform worse with the exception of strategy 2-32, which is very similar to $1-32$ and survives in 10 out of $12(83 \%)$ of the simulations which it was involved in. In these 25 additional simulations the average number of points increases and the average propensity to change decreases over generations, like in Figure 5 for the original evolutionary simulations. The average number of points in generation 491-500 is slightly less than in the original simulations, 37.12 versus 38.40 , and the average propensity to change is higher, $12 \%$ versus $8 \%$.

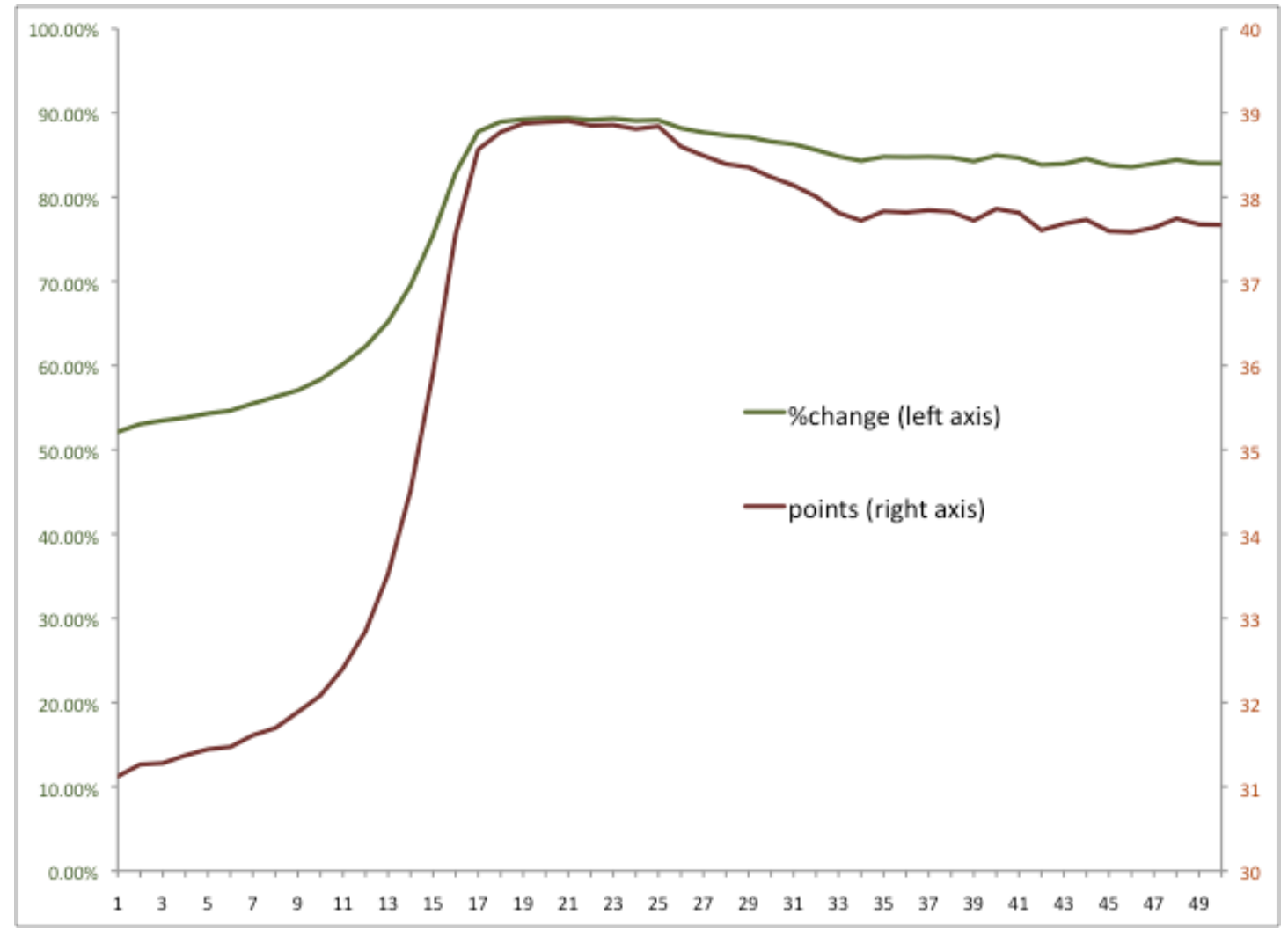

Figure 6: Evolutionary simulations with strategies of cluster $5 / 6$ and $6 / 6$ excluded. On the left axis change (green line) and on the right axis (red) earnings.

The evolutionary simulations that we discussed this far all suggest that as evolution proceeds the surviving strategies will change color less, on average. This, of course, also has to do with the environment: in an environment where other strategies change more often than not, a good strategy might be also to change more often. To illustrate this point we have run 5 simulations with the strategies of clusters $5 / 6$ and $6 / 6$ excluded. In the first generation the average number of changes is about $52 \%$; which increases to about $85 \%$ in generation 50 and more (see Figure 6). Also the average earnings increase: to an impressive 39 points around generation 20 and finally stabilizing around 37.8 points. 


\section{Conclusion}

In this paper we used an internet based strategy method experiment to explicitly elicit the strategies employed in the minority game. Participants could try out their strategies on the minority game website by simulating them against strategies submitted by the other participants in the previous round. This allowed them to adapt their strategies in the direction they believe will be successful. We believe this is not only a novel aspect of the experimental design, but also relevant for many applications where decision makers, for example traders in financial markets, have the possibility to employ technological tools to try to improve their decisions.

We find that the aggregate behavior of the submitted strategies is close to that implied by the symmetric mixed strategy Nash equilibrium. However, there is considerable heterogeneity between the submitted strategies. Remarkably, participants do not structurally succeed in improving their strategies over the five rounds, and hence the amount of learning seems to be limited, although the minority game website provides ample opportunities to learn.

A cluster analysis revealed that the submitted strategies can be divided in six distinct groups. The central strategies in each cluster give an idea of the types of strategies in each group. We find both "lose shift" (1/6) and "win shift" (4/6) types of strategies. The most central strategy in another cluster (6/6) also only changes after losing, but with a low probability. The central strategies of the three other clusters are independent of the history and either change very often (1/1), half the time as in the symmetric mixed strategy Nash equilibrium (3/6), or never (6/6). Success of a strategy is negatively correlated with how often the strategy changes sides. The most successful strategies come from cluster 5/6 and have a tendency to only change sides after being on the losing side for two consecutive periods or more.

We find that people use mixed strategies and condition their actions on more detailed histories than just winning or losing. This shows that the restrictions placed on strategies in many evolutionary simulations of the minority game do not allow for the kind of strategies people actually use. We therefore perform an evolutionary simulation with the 107 unique strategies gathered in the experiment.

Although the most successful strategies from the actual strategy experiment perform relatively well in the evolutionary competition, they do die out eventually, to the benefit of some other strategies that are slightly less reluctant to change. The 
intuition for this is that the initially successful strategies do quite well in an environment where all submitted strategies are present, because they profit from strategies that change often. When the latter strategies become extinct, however, the initially successful strategies are not very well suited to the new strategic environment and strategies which enhance efficiency take over. The surviving strategies are reluctant to change, which enhances stability, but they do change, with a small probability, especially in inefficient situations. Evolutionary competition therefore leads to a fast and dramatic improvement in coordination.

The impressive increase of coordination in the evolutionary simulation contrasts starkly with the lack of increase in coordination during the experiment. This difference suggests that participants on average fail to improve their strategies between rounds despite the possibilities offered by the minority game website. As a result there is very little coordination and aggregate outcomes resemble the mixed strategy equilibrium. Our experiment and the evolutionary simulation with the gathered strategies therefore suggest increasing learning is the key to improving coordination in the minority game. We aim to examine ways to increase learning in future experiments.

\section{Appendix: Instructions and Screenshots Minority Game Experiment (translated from Dutch)}

\section{Game instructions}

The game is played with 5 players. All these players choose between red or blue. Players who selected the color selected by the smallest number of players earn one point. The other players earn nothing.

In the experiment the decision isn't made directly by you, but by a strategy devised by you. This strategy decides when you change color and when you don't. How this exactly works is explained below.

We will first play the game for a number of rounds where you do take the decision yourself. The first round you choose red or blue. In later rounds you choose whether to change color or not.

PARTICIPANTS THEN TWICE PLAYED THE MINORITY GAME FOR 10 ROUNDS AFTER WHICH THEY RECEIVED THE FOLLOWING INSTRUCTIONS

\section{Instructions experiment}

\section{Strategies}

Before you decided each round whether to change color or not. From now on you will formulate a strategy which in each situation decides for you whether to change color or not. You specify in which situations you want to change color. You hand in this strategy in the form of a computer code. How this works is explained below.

\section{Conditions}

We use computer code consisting of so called "IF statements" that look like this: “ 
IF (condition) \{ RETURN number ;" $\}$ “.

With these you can determine when you will change or not. Your strategy can consist of multiple if statements.

\section{Condition}

The condition in your if statement is either true or false. In the condition you can use the history of the previous 5 rounds: per period the number of players with your color (including you) and whether you changed color. The table below shows the codes for these events. During the experiment you can make strategies by clicking on the required codes, so you do not need to learn the codes by heart. In construction your conditions you can use arguments. These arguments are: and/or (OR), and (AND), negation (!), equality (=), smaller than ( $<$ ), larger than ( $>)$, brackets (). You can use these arguments by clicking on them. To use the arguments $==,>$ and $<$ you should view the events as variables which have the value 1 if they are true and 0 if they are false. You can add or subtract conditions using + and -.

\begin{tabular}{|c|c|c|c|c|c|c|}
\hline IF statement & AND OR & () & gation! & $==$ & $<+$ & - clea \\
\hline & ChonodL & & nber of $\mathrm{pl}$ & yers with & our col & \\
\hline & |Cranget| & & & & Lose & \\
\hline Premious round. & $40[11]$ & & [1] & & Lose[1] & J \\
\hline 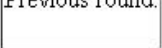 & \$C[I] & $\$ \mathrm{~W} 1[1]$ & \$W/2[1] & $\$$ \$L3[1] & $\$$ & $\$\llcorner 5[1]$ \\
\hline 2 rounds ara. & 5001 & & [2] & & Lose[2] & $\square$ \\
\hline & & \$W1[2] & $\$ W 2[2]$ & 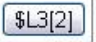 & $\$ L 4[2]$ & $\$\llcorner 5[2]$ \\
\hline & $9 c 131$ & & & & Lose[3] & $\square$ \\
\hline 3 rounds ago: & $s<[3]$ & $\$ W 1[3]$ & \$W2[3] & $\$ \$ \$ 3[3]$ & \$L4[3] & $\$$ \\
\hline & & & & & LLose[4] & ] \\
\hline 4 rounds ago: & $\$ C[4]$ & $\$ W 1[4]$ & $\$ W 2[4]$ & 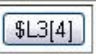 & $\$\llcorner 4[4]$ & $\$\llcorner 5[4]$ \\
\hline 5 rounds ano & of 1 1 & & & & LLose[5] & $\square$ \\
\hline P rounias ago. & s[l5] & \$W1[5] & $\$ W 2[5]$ & $\$ 153[5]$ & $\$\llcorner 4[5]$ & $\$ L 5[5]$ \\
\hline
\end{tabular}

(this is an example, you can not yet click on anything.)

Below you will find a number of examples of IF statements. These are only examples and not necessarily smart strategies.

Example 1 (OR argument)

IF (\$W1[2] OR \$C[5])

means "if I won 2 periods ago with 1 player (including myself) choosing my color and/or if I changed color 5 periods ago."

Example 2 (AND argument and negation!)

IF (\$L3[4] AND ! \$C[2])

means "if I lost 4 periods ago with 3 players (including myself) choosing my color and I did not change 2 periods ago."

Example 3(inequality $>$ )

IF $(\$ C[1]+\$ C[2]+\$ C[3]>\$ W 2[1]+\$ W 2[2]+\$ W 2[3])$

means "if $\mathrm{I}$ in the previous 3 periods changed color more often than I won with 2 players (including myself) choosing my color in those same periods "

Example 4(equality == and negation !)

IF $(\$ C[3]==$ ! \$W2[5])

means "if I changed 3 periods ago and I did not win with 2 players (including myself) choosing my color 5 periods ago or if I did not change 3 periods ago and I did win with 2 players (including myself) choosing my color 5 periods ago."

\section{Number}

Your IF statement always ends with " $\{$ RETURN getal ; $\} "$. The number you fill out here determines 
what happens if your condition is true. A 1 means you will change color, a 0 that you will not and a number between 0 and 1 means that you will change color with that probability.

Example 5 (number between 0 en 1)

IF $(\$ L 5[4]==\$ W 2[1])\{$

RETURN 0.64;

\}

means "if I lost 4 periods ago with 5 players (including myself) choosing my color and I won 1 period ago with 2 players (including myself) choosing my color, or both are not true, I will change color with a probability of $64 \%$. "

You can also use 1 as a condition. 1 means "always true".

Example 6 (condition that is always true: 1)

IF (1) \{

RETURN 0.4;

\}

means "independent of the history I will change color with a probability of $40 \%$."

\section{Strategy}

Your strategy can consist of multiple IF statements. In that case the statements are reviewed in the order in which you wrote them down. If a condition in an IF statement is true, subsequent IF statements are ignored. (For those with programming experience: they can be considered ELSEIF statements). If non of your IF statements is fulfilled it is assumed that you will not change color.

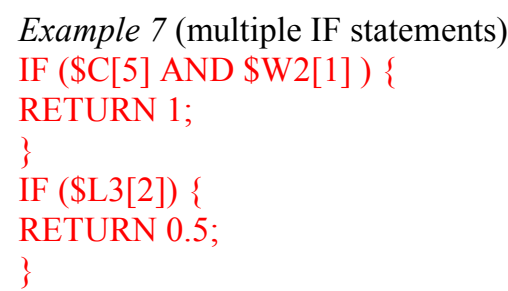
color in the previous period, I will change color. If that is not true, but I have lost with 3 players (including myself) choosing my color 2 periods ago I will change color with a $50 \%$ probability. Otherwise I do not change."

Example 8 (multiple IF statements) IF (\$W1[4] OR (\$C[1] AND \$L4[3] ) ) color in the previous period and I have lost with 4 players (including myself) choosing my color 3 periods ago, than I change color with a probability of $20 \%$. In all other cases I change color with a probability of $70 \%$."

During the experiment you can either click on all the codes you may need while making a strategy or write them down yourself. You can also cut ( $\operatorname{ctrl} x)$, copy (ctrl c), paste (ctrl v) and undo things ( $\operatorname{ctrl} z)$, or redo things that you undid (ctrl y).

\section{Simulations and results}

Participants earnings in every round depended on the place of their strategy in the ranking of strategies. In order to determine a ranking of strategies all possible combinations of strategies are considered in a simulation.

Each simulation starts with 5 rounds where each players chooses red or blue with equal chance. This way a random history is created. Then 100 rounds are played with the same combination of strategies. For the history it is assumed that you didn't change color in the first round. The 5 random rounds don't 
count towards a strategies score.

For each simulation the number of points scored by each strategy is recorded. The final score is the average score over all simulations a strategy was involved in. On this basis a ranking is determined. Using this ranking earnings were determined according to the following table:

\begin{tabular}{|c|c|c|}
\hline & Rounds $1,2,3$ and 4 & Round 5 \\
\hline Best strategy & $€ 75$ & $€ 150$ \\
\hline Second place & $€ 60$ & $€ 120$ \\
\hline Third place & $€ 45$ & $€ 90$ \\
\hline Fourth place & $€ 30$ & $€ 60$ \\
\hline Fifth place & $€ 15$ & $€ 30$ \\
\hline All other strategies & $€ 0$ & $€ 0$ \\
\hline
\end{tabular}

\section{New strategy}

After each round you receive the results by email. In your email you will find the webaddress to adjust your strategy for the next round.

After each round you can see how your strategy performs by seeing what happens when your strategy plays against four random strategies from the previous round. You can do this as often as you want. Than you can change your strategy (of course you do not have to). You can also try new strategies against random strategies from the previous round. When you are satisfied with your new strategy you hand it in. You can also hand in your old strategy. If you do not hand in any strategy, you can not make any money.

\section{Questionnaire}

After handing in a strategy you will be presented with a questionnaire. For each round in which you fill out the questionnaire you will receive 5 euros.

\section{PARTICIPANTS WERE THEN ASKED TO PROGRAM TWO VERBAL STRATEGIES TO TEST THEIR UNDERSTANDIN OF WRITING A STRATEGY. AFTER THEY SUCCESFULLY PROGRAMED THESE STRATEGIES THEY RECEIVED THE FOLLOWING INSTRUCTIONS BEFORE THEY PROGRAMMED THEIR FIRST STRATEGY.}

You are about to program a strategy. You can check this strategy in a simulation. In this simulation you can not see how good your strategy is because the other strategies are selected more or less randomly by us and may be different in nature than the strategies of your fellow students against which you will compete.

You can use the simulation to check whether your strategy behaves as intended and you did not make a mistake programming it.

If the simulation shows that your strategy does not behave as you intended you can adjust it and run a new simulation.

When you click on the "this is my strategy" button this is your strategy for the first round. Only when you receive the results per email can you change your strategy for round two.

PARTICIPANTS ALSO RECEIVED A SUMMARY OF THESE INSTRUCTIONS ON PAPER AND COULD READ THE INSTRUCTIONS ON THE WEBPAGE USED TO PROGRAM, TEST AND HAND IN STRATEGIES FOR ROUNDS TWO TO FIVE. 
Example of a screen to formulate a strategy (translation from Dutch). The buttons had context dependent help (showed in the red box).

\section{Formulating the strategy}

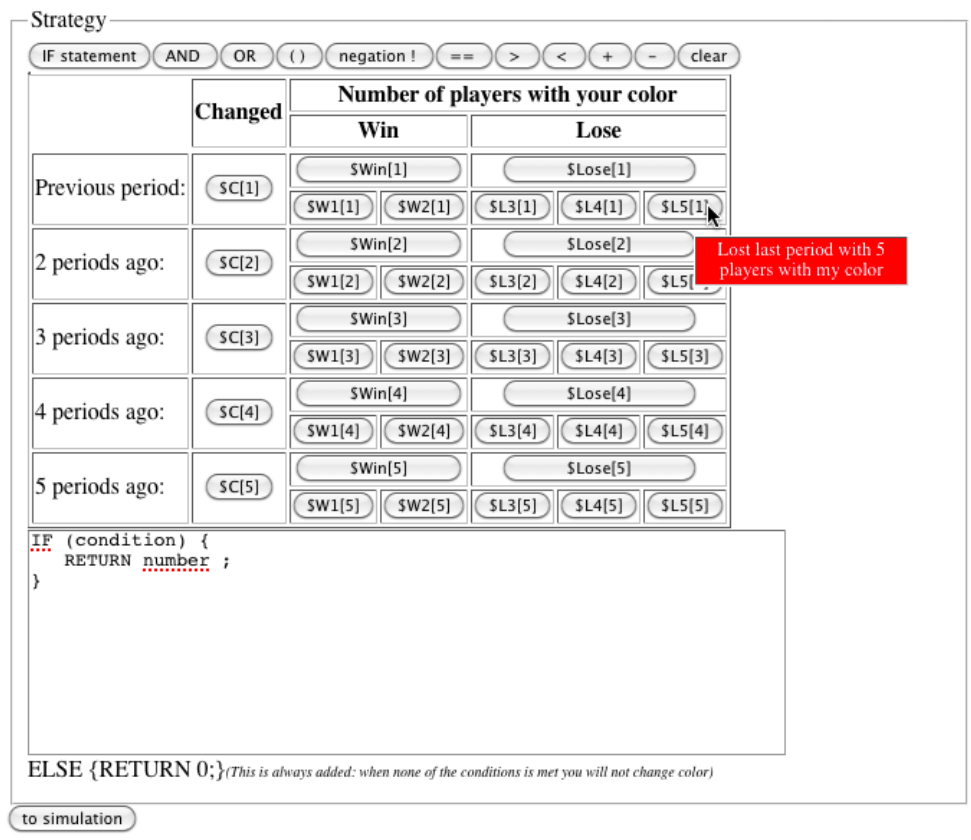

Click here to view the complete instructions.

Example of a screen after a strategy is tried out by the participant (translated from Dutch).

Simulations

with strategy:

IF (\$LA $[1])\{$

RETURN .2;

\}

IF (\$L5]1]) \{

RETURN 3;

ELSE \{RETURN 0;\}

\begin{tabular}{|c|c|c|c|c|}
\hline Period & Self & Others & Changed & Result \\
\hline & & & & \\
\hline 101 & B & $R R R B$ & 0 & W2 \\
\hline 102 & B & $R R B B$ & 0 & L3 \\
\hline 103 & B & $R R B B$ & 0 & L3 \\
\hline 104 & B & $R R B B$ & 0 & L3 \\
\hline 105 & B & $R R R R$ & 0 & W1 \\
\hline
\end{tabular}

\begin{tabular}{|c|c|c|c|}
\hline \multicolumn{4}{|c|}{ Results 100 periods } \\
\hline Win & Lose & & \\
\hline W1 W2 & L3 $\overline{\mathrm{L} 4}$ & L5 & Points \\
\hline 26 & \begin{tabular}{|l|l|}
38 & 31 \\
\end{tabular} & 1 & 30 \\
\hline
\end{tabular}

New simulation

Other strategy

This is my strategy! (To submit the definite strategy for that period, not working in the demo)

If you do not want to test any other strategies at this time and do not want to register a final strategy you can log out. Do not forget to register a strategy before the deadline, otherwise you can not make money during this round.

Log out 


\section{Example feedback of round by email (translated from Dutch).}

Dear [name participant]

The simulations for round [round number] are finished and these are the results:

Your strategy finished on place [rank] and your earnings in this round are [earnings].

The general results

$\begin{array}{lll}\text { Rank } & \text { Login name } & \text { Average number of points } \\ 1 & \text { Chung-Lin KWA! } & 39.873644 \\ 2 & \text { gemer92 } & 38.148873 \\ 3 & \text { Kees } & 37.256570 \\ 4 & \text { Witchy } & 36.612166 \\ \ldots . & & \\ 35 & \text { A-town } & 27.996524 \\ 36 & \text { capital P } & 27.888102\end{array}$

After logging in on www.creedexperiment.nl/minor/login.php you can run simulations with your strategy of round [round number] and 4 other strategies also from round [round number]. After that you can try out new strategies against 4 strategies of round [round number] and after that submit your final strategy for round [round number +1 ].

Best regards,

Jona Linde, Joep Sonnemans en Jan Tuinstra

\section{References}

Arthur, W.B. (1994). Inductive reasoning and bounded rationality. American Economic Review 84, 406-411.

Axelrod, R. (1984). The Evolution of Cooperation. New York: Basic Books.

Bottazzi, G. and G. Devetag (2003). A laboratory experiment on the minority game. Physica A 324, 124-132.

Bottazzi, G. and G. Devetag (2007). Competition and coordination in experimental minority games. Journal of Evolutionary Economics 17, 241-275.

Brandts, J. and G. Charness (2011). The strategy versus the direct response method: a first survey of experimental comparisons. Experimental Economics 14, 375-398

Cavagna, A., J.P. Garrahan, I. Giardina and J. Sherrington (1999). Thermal model for adaptive competition in a market. Physical Review Letters 83, 4429-4432.

Challet, D., M. Marsili, and Y.-C. Zhang (2000a). Modelling market mechanism with minority game. Physica A 276, 284-315.

Challet, D., M. Marsili, and Y.-C. Zhang (2001). Stylized facts of financial markets and market crashes in minority games. Physica A 294, 514-524.

Challet, D., M. Marsilli, and R. Zecchina (2000b). Statistical mechanics of systems with heterogeneous agents: Minority games. Physical Review Letters 84, $1824-$ 1827.

Challet, D. and Y.-C. Zhang (1997). Emergence of cooperation and organization in an evolutionary game. Physica A 246, 407-418.

Challet, D. and Y.-C. Zhang (1998). On the minority game: Analytical and numerical studies. Physica A 256, 514-532.

Chmura, T., W. Guth, T. Pitz and A. Ziegelmeyer (2010). The minority of threegame: an experimental and theoretical analysis. Jena Economic Research Paper 2010-071,

Chmura, T. and T. Pitz (2006). Succesful strategies in repeated minority games. Physica A 363, 477-480. 
Devetag, G., F. Pancotto and T. Brenner (2011). The minority game unpacked: coordination and competition in a team-based experiment. University of Bologna Working Paper DSE 770.

Duffy, J. and E. Hopkins (2005). Learning, information, and sorting in market entry games: theory and evidence. Games and Economic Behavior 51, 31-62.

Erev, I. and A. Rapoport (1998). Coordination, "Magic," and Reinforcement Learning in a Market Entry Game. Games and Economic Behavior 23, 146-175.

Ezekiel, M. (1938). The cobweb theorem. Quarterly Journal of Economics 52, 255 280.

Fernández, A. and S. Gómez (2008). Solving non-uniqueness in agglomerative hierarchical clustering using multidendrograms. Journal of Classification 25, 43-65.

Hommes, C., J. Sonnemans, J. Tuinstra and H. van de Velden (2005). A strategy experiment in dynamic asset pricing. Journal of Economic Dynamics and Control 29, 823-843.

Huberman, B.A. and R.M. Lukose (1997). Social dilemmas and internet congestion. Science 277, 535-537.

Iida, Y., T. Akiyama and T. Uchida (1992). Experimental analysis of dynamic route choice behavior. Transportation Research B 26B, 17-32.

Johnson, N.F., P.M. Hui, R. Jonson and T.S. Lo (1999). Self-organized segregation within an evolving population. Physical Review Letters 82, 3360-3363.

Leady, J.R. (2000). Learning in coordination games utilizing categorized information: Experimental evidence. Unpublished Memo University of Michigan.

Li, Y., R. Riolo and R. Savit (2000a). Evolution in minority games. (I). Games with a fixed strategy space. Physica A 276, 234-264.

Li, Y., R. Riolo and R. Savit (2000b). Evolution in minority games. (II). Games with variable strategy spaces. Physica A 276, 265-283.

Platkowski, T. and M. Ramsza (2003). Playing minority game. Physica A 323, 726734.

Rapoport, A., D.A. Seale, I. Erev and J.A. Sundali (1998). Equilibrium play in large group market entry games. Management Science 44, 119-141.

Renault, J., S. Scarlatti and M. Scarsini (2005). A folk theorem for minority games. Games and Economic Behavior 53, 208-230.

Rosenthal, R.W. (1973). A class of games possessing pure-strategy Nash equilibria. International Journal of Game Theory 2, 65-67.

Savit, R., R. Manuca and R. Riolo (1999). Adaptive competition, market efficiency, and phase transitions. Physical Review Letters 82, 2203-2206.

Seale, D.A. and A. Rapoport (2000). Elicitation of strategy profiles in large group coordination games. Experimental Economics 3, 153-179.

Selten, R., T. Chmura, T. Pitz, S. Kube, M. Schreckenberg, (2007). Commuters route choice behaviour, Games and Economic Behavior, 58, 394-406.

Sonnemans, J., C. Hommes, J. Tuinstra and H. van de Velden (2004). The instability of a heterogeneous cobweb economy: A strategy experiment on expectation formation. Journal of Economic Behavior and Organization 54, 453-481.

Sundali, J.A., A. Rapoport and D.A. Seale (1995). Coordination in market entry games with symmetric players. Organizational Behavior and Human Decision Processes 64, 203-218.

Sysi-Aho, M., A. Chakraborti and K. Kaski (2005). Searching for good strategies in adaptive minority games. Physical Review E 69, 036125 
Szekely, G.J. and M.L.Rizzo (2005). Hierarchical clustering via joint between-within distances: Extending Ward's minimum variance method. Journal of Classification 22, 151-183.

Wang, W., Y. Chen and J. Huang (2009). Heterogeneous preferences, decisionmaking capacity, and phase transitions in a complex adaptive system. Proceedings of the National Academy of Sciences 106, 8423-8428.

Yip, K.F., P.M. Hui, T.S. Lo and N.F. Johnson (2003). Efficient resource distribution in a minority game with a biased pool of strategies. Physica A 321, 318-324.

\section{Acknowledgements}

We thank the Research Priority Area Behavioral Economics of the University of Amsterdam for financial assistance and participants of seminars in Amsterdam, Nottingham, Konstanz and Innsbruck and participants to CEF2010 (London), ABEE2011 (Amsterdam), the 2012 Asian-pacific ESA conference (Xiamen) and the 2012 International ESA Conference (New York) for useful comments. 